\title{
Evaluating the Usability and Acceptability of the HARP Mobile App
}

Nicole Polanco ${ }^{1 *}$; Ramya Palacholla ${ }^{1,2,3^{*}}$; Tasmia Noor ${ }^{1 *}$; Jung-Taek $\mathrm{Oh}^{4 *}$

${ }^{1}$ Partners Healthcare, Boston, MA, United States

${ }^{2}$ Massachusetts General Hospital, Boston, MA, United States

${ }^{3}$ Harvard Medical School, Cambridge, MA, United States

${ }^{4}$ UrCare, Cambridge, MA, United States

*all authors contributed equally

\section{Corresponding Author:}

Jung-Taek Oh

UrCare

1 Broadway St

Cambridge, MA

United States

Phone: 6175050037

Email: jt.oh@e2ehealth.com

\begin{abstract}
Background: Studies show that good communication between doctors and patients and among all caregivers who interface with patients directly results in better clinical outcomes, reduced costs, greater patient satisfaction, and lower rates of physician burnout. The main purpose of this pilot study was to test the acceptability and usability of a mobile phone app (HARP), an app designed to improve communication and data collection among nonclinical care givers such as home health aides and case managers of patients who receive care at home. The home health aides collected information on patient's mood, energy, medication adherence, potential falls and appetite level for the day. This information is summarized in postvisit, weekly and final discharge summary reports via an online dashboard and sent to the patient's case manager at different time points. We assessed the usability and acceptability of the HARP mobile app.
\end{abstract}

Objective: This is a quality improvement pilot project geared towards assessing the usability and acceptability of a mobile app developed to facilitate patient data collection by trained home health aides who work together on a regular basis to provide home-based care to discrete subpopulations of patients.

Methods: Four home health aides were recruited from Partners Healthcare at Home to use the app to collect data on at least 12 patients. Eligible patients received care from 1 of the 4 home health aides for at least 23 days and scheduled to have at least 5 home visits during this time. Each of the patients were followed for a minimum of 23 days and a maximum 60 days in which home health aides collected patient data using the app. Postvisit reports, weekly reports and discharge summary reports were shared with the patient's case managers. Data collection included acceptability and satisfaction data from all home health aides and case managers via surveys. A subgroup of 2 case managers and 2 home health aides participated in semistructured interviews.

Results: 9 have completed the project to date, 5 patients dropped out due to discharge from Partners Healthcare at Home care. The interim data included is from 8 case managers and 4 home health aides who provide care to one or more of the 9 patients who completed the project. Most case managers (75\%) found postvisit and weekly reports useful and $87 \%$ found tracking mood and energy helpful. About 75\% felt tracking appetite and falls via the HARP app helpful. Almost all case managers (87\%), agreed that integrating a tool like the HARP app to the EMR would help them provide better care to their patients. Three out of four home health aides $(75 \%)$ felt that the app easy to use or learn about once they received instructions and were willing to consider using the app in their workflow.

Conclusions: Acceptability and usability of HARP app was considerably high among case managers. The acceptability of the app varied among home health aides, some found information useful and believed the app has potential to help personalize patient care. Future research would require exploring other patient information that is useful to all staff involved in the clinical workflow and increase adoption of tool in clinical settings. Such tools could potentially reduce clinician burnout and improve patient outcomes. 
(iproc 2019;5(1):e16256) doi: $10.2196 / 16256$

\section{KEYWORDS}

mobile app; health; home care

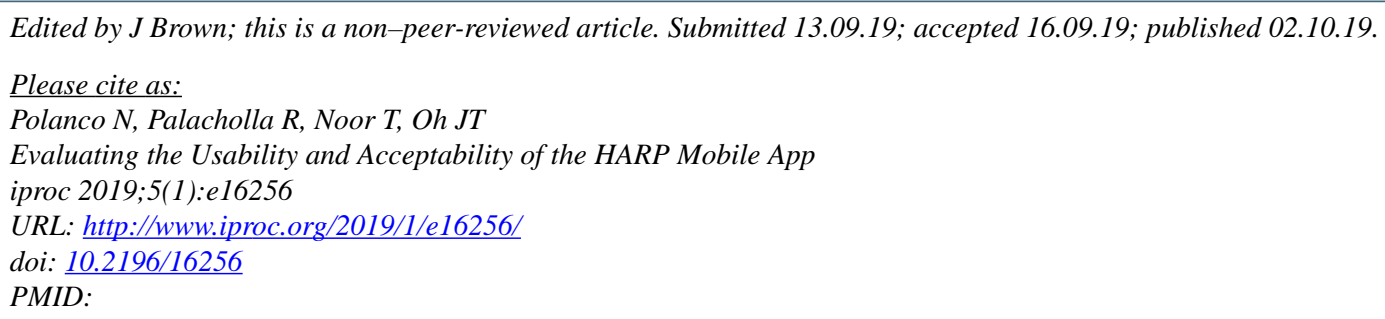

CNicole Polanco, Ramya Palacholla, Tasmia Noor, Jung-Taek Oh. Originally published in Iproceedings (http://www.iproc.org), 02.10.2019 This is an open-access article distributed under the terms of the Creative Commons Attribution License (https://creativecommons.org/licenses/by/4.0/), which permits unrestricted use, distribution, and reproduction in any medium, provided the original work, first published in Iproceedings, is properly cited. The complete bibliographic information, a link to the original publication on http://www.iproc.org/, as well as this copyright and license information must be included. 